\title{
ON THE EXTENSION OF INTERVAL FUNCTIONS
}

\author{
BY \\ LAWRENCE A. RINGENBERG
}

Introduction. The problem of extending the range of definition of a function defined on a class of elementary figures-intervals, rectangles-has been treated in various ways in the literature. In the theory of Lebesgue measure a particular function-length of interval (area of rectangle)-is extended in a completely additive way to an additive class of sets. In the general extension problem we start, say, with a function (real, single-valued, and finite) of intervals $\phi(I)$ and extend the range of definition to an additive class of sets obtaining a function $\Phi(E)$ which is completely additive and which has the property that $\Phi(E)=\phi(I)$ whenever " $E$ is the set $I$." But what is the interval $I$ ? A priori $\phi(I)$ is defined on a class of intervals $I$, where $I$ is considered neither open nor closed but merely as an interval. From the viewpoint of $\Phi(E)$ an interval $I$ must be considered as a definite point set-a closed interval, an open interval, a semi-open interval, and so on. Corresponding to open intervals and to closed intervals, $\Phi(E)$ gives rise to two interval functions: $\phi_{1}(I)=\Phi\left(I^{\prime}\right), \phi_{2}(I)=\Phi\left(I^{0}\right)$ where $I^{\prime}$ is understood to be closed and $I^{0}$ open. If $\phi(I)=\phi_{1}(I)$ identically, then $\Phi(E)$ is an extension of $\phi(I)$ considered as a function of closed intervals; if $\phi(I)=\phi_{2}(I)$ identically, then $\Phi(E)$ is an extension of $\phi(I)$ considered as a function of open intervals. As a starting point in the general extension problem, the function $\phi(I)$ has been considered, somewhat artificially and arbitrarily perhaps, a function either of closed intervals or of open intervals (see, for example, [10]) ${ }^{1}$ ). Extensions $\Phi(E)$ which have the property that $\Phi\left(I^{\prime}\right)=\Phi\left(I^{0}\right)$ identically are of particular interest since then $\Phi(E)$ is an extension of $\phi(I)$ whether $I$ be considered open or closed.

The main results of the paper concern the existence of $B$-extensions, a precise definition of which is given in $\$ 1.6$. Suffice it to say here that if $\Phi(E)$ is a $B$-extension of $\phi(I)$ then $\Phi\left(I^{\prime}\right)=\Phi\left(I^{0}\right)=\phi(I)$. The idea of a $B$-extension was suggested by a result of Burkill [2] which we shall review in $\S 1.5$. Burkill's theorem on extension is stated in terms of a sufficient condition while our results on $B$-extensions are stated in terms of necessary and sufficient conditions.

In Part 1 we explain notation, define terms, and summarize results. In Part 2 we present a proof of a theorem (Theorem 1) which states a necessary and sufficient condition that a non-negative function of closed intervals

Presented to the Society, April 27, 1946; received by the editors August 6, 1941, and, in revised form, March 13, 1946.

(1) Numbers in brackets indicate references in the bibliography at the end of the paper. 
admit a non-negative completely additive extension. This theorem was proved in [10] using the results of Radon [9]. The present proof makes use of the theory of outer measure in the sense of Carathéodory. Theorem 2 extends the result of Theorem 1 to the case of a function of arbitrary sign. Theorem 3 concerns the uniqueness and characterization of an extension of a function of intervals. Part 3 contains proofs of our results (Theorems 4,5 , and 6 ) on the $B$-extension. We present necessary and sufficient conditions that a non-negative function of intervals, a function of intervals of arbitrary sign, and an indefinite integral of a function of intervals, respectively, admit $B$-extensions.

\section{Preliminaries and summary.}

1.1. In modern literature intervals are considered as $k$-dimensional where $k$ is a positive integer. We shall consider functions of intervals in the $x y$-plane, that is, our intervals are two-dimensional. The word interval is used in the sequel only in the accepted point set sense: Given two points $\left(x_{1}, y_{1}\right),\left(x_{2}, y_{2}\right)$ an interval is the set of points $(x, y)$ such that $x_{1} \leqq x \leqq x_{2}, y_{1} \leqq y \leqq y_{2}$; an open interval is the set of points $(x, y)$ such that $x_{1}<x<x_{2}, y_{1}<y<y_{2}$ (Saks [12, p. 57$]$ ). Let $R_{0}$ be the interval $0 \leqq x \leqq 1,0 \leqq y \leqq 1$. All sets considered in this paper are subsets of $R_{0}$ unless otherwise stated. We use the letters $I, J, R$ to denote intervals. The symbol $I^{0}$ denotes the open interval which corresponds to $I$, that is, $I^{0}$ is the interior of the set $I$. The letter $C$ is used to denote a class of intervals. A capital script letter, as $\mathcal{E}$, is used to denote an elementary system of intervals, that is, a finite set of intervals $I_{1}, I_{2}, \cdots, I_{k}$ such that $I_{i}^{0} \cdot I_{j}^{0}=0$ whenever $i \neq j$. A capital script letter is also used as an operator in the sense that $\varepsilon_{I}$ denotes an elementary system of intervals $I_{1}, I_{2}, \ldots i, I_{k}$ which constitutes a subdivision of $I$, that is, $\sum_{i=1}^{k} I_{i}=I$. The parameter of regularity of an interval $I$, denoted by $p(I)$, is the ratio of the length of the shorter side of $I$ to the length of the longer side of $I$. The norm of an interval $I$, denoted by $\|I\|$, is the length of the diameter of $I$; the symbol $\|\varepsilon\|$ is defined as the largest of the numbers $\|I\|$ where $I \in \mathcal{E}$. The measure of an interval $I$ is denoted by $|I|$; the symbol $|\varepsilon|$ is defined as the number $\sum|I|$ where the sum is taken over $I \in \mathcal{E}$. The boundary of an interval $I$, denoted by $b(I)$, is the set $I-I^{0}$.

1.2. A class of sets in $R_{0}$ is said to be closed (relative to $R_{0}$ ) - and is generically denoted by $K$-if the following conditions are satisfied:

(i) Every set open relative to $R_{0}$ (denoted generically by $O$ ) is in $K$.

(ii) If a set $E$ is in $K$, then the complement of $E$ relative to $R_{0}$ (denoted by $C E$ ) is also in $K$.

(iii) If $\left\{E_{n}\right\}$ is a sequence of sets in $K$, then $\sum_{n} E_{n}$ is also a set in $K$.

Clearly every closed class $K$ contains all Borel sets $E \subset R_{0}$. In fact, the class of all Borel sets in $R_{0}$, which we denote by $B$, is identical with the product of all closed classes in $R_{\mathbf{0}}$.

Let $\lambda$ be a fixed number satisfying the relation $0 \leqq \lambda<1$, and let $C_{\lambda}$ denote the class of all intervals $I$ such that $p(I) \geqq \lambda$. A subscript $\lambda$ as in $I_{\lambda}$ and $\varepsilon_{\lambda}$ 
indicates that $I_{\lambda} \in C_{\lambda}$ and that $I \in C_{\lambda}$ for every $I \in \varepsilon_{\lambda}$. The symbol $C_{0}$ will denote the class of all intervals $I$ such that $I \subset R_{0}$. Let $\phi(I)$ denote a real, finite, single-valued function which is defined for every $I \in C_{\lambda}$. This function is denoted briefly by the symbol $\left[\phi, C_{\lambda}\right]$ in which the first letter denotes the function and the second letter denotes the range of definition of the function.

A function $\Phi(E)$ which is defined on a closed class $K$, that is, the function $[\Phi, K]$, is a completely additive extension of the function $\left[\phi, C_{\lambda}\right]$ if the following conditions are satisfied:

(i) $[\Phi, K]$ is a completely additive set function.

(ii) $\Phi(I)=\phi(I)$ for every $I \in C_{\lambda}$.

In Part 2 we shall prove the following theorems:

ThEOREM 1. (See [10, Theorem 3].) A necessary and sufficient condition that a non-negative function of intervals $\left[\phi, C_{\lambda}\right]$ have a non-negative completely additive extension is that it satisfy the following condition $\mathbb{E}:$ If $\left\{I_{n}\right\}$ is any sequence of intervals in $C_{\lambda}$ such that $I_{i} \cdot I_{j}=0$ when $i \neq j$ and if $\left\{J_{m}\right\}$ is any sequence of intervals in $C_{\lambda}$ such that $\sum_{m} J_{m} \supset \sum_{n} I_{n}$ then $\sum_{m} \phi\left(J_{m}\right) \geqq \sum_{n} \phi\left(I_{n}\right)$.

THEOREM 2. A necessary and sufficient condition that a function of intervals $\left[\phi, C_{\lambda}\right]$, of arbitrary sign, have a completely additive extension is that it be the difference of two non-negative functions each of which satisfies condition $\mathfrak{5}$.

THEOREM 3. If $[\Phi, K]$ is a completely additive extension of a function of intervals $\left[\phi, C_{\lambda}\right]$, then the value of the number $\Phi(E)$, where $E$ is any Borel set, is uniquely determined by the function $\left[\phi, C_{\lambda}\right]$. If $\left[\phi, C_{\lambda}\right]$ is non-negative, then $[\Phi, K]$ is also non-negative and for every Borel set $E$ we have the characterization: $\Phi(E)=$ g.l.b. $\sum_{n} \phi\left(I_{n}\right)$ for all sequences $\left\{I_{n}\right\}$ such that $\sum_{n} I_{n} \supset E$ and $I_{n} \in C_{\lambda}$, $n=1,2, \cdots$.

1.3. Given a function of intervals $\left[\phi, C_{\lambda}\right]$, we extend the range of definition of $\left[\phi, C_{\lambda}\right]$ to include all elementary systems of intervals $\varepsilon_{\lambda}$ as follows: $\phi\left(\varepsilon_{\lambda}\right)=\sum \phi(I)$ where the sum is taken over $I \in \varepsilon_{\lambda}$. The function $\left[\phi, C_{\lambda}\right]$ is additive if for every $I_{\lambda}$ and every $\varepsilon_{\lambda} I_{\lambda}$ it is true that $\phi\left(E_{\lambda} I_{\lambda}\right)=\phi\left(I_{\lambda}\right)$. If we replace $\phi\left(\varepsilon_{\lambda} I_{\lambda}\right)=\phi\left(I_{\lambda}\right)$ by $\phi\left(\varepsilon_{\lambda} I_{\lambda}\right) \geqq \phi\left(I_{\lambda}\right)$ and then by $\phi\left(\varepsilon_{\lambda} I_{\lambda}\right) \leqq \phi\left(I_{\lambda}\right)$ we obtain the definitions of a function which increases by subdivision and decreases by subdivision respectively. The function $\left[\phi, C_{\lambda}\right]$ is continuous if for every number $\epsilon>0$ there exists a number $\delta>0$ such that (i) $\left|I_{\lambda}\right|<\delta$ implies $\left|\phi\left(I_{\lambda}\right)\right|<\epsilon$ and (ii) $I_{\lambda 1} \subset I_{\lambda 2},\left|I_{\lambda 2}-I_{\lambda 1}\right|<\delta$ imply $\left|\phi\left(I_{\lambda 1}\right)-\phi\left(I_{\lambda 2}\right)\right|<\epsilon$. It is observed that condition (ii) in this definition is a consequence of condition (i) if the function is additive and $\lambda=0$. The function $\left[\phi, C_{\lambda}\right]$ is absolutely continuous if for every number $\epsilon>0$ there exists a number $\delta>0$ such that $\left|\varepsilon_{\lambda}\right|<\delta$ implies $\left|\phi\left(\varepsilon_{\lambda}\right)\right|<\epsilon$.

1.4. Given a function of intervals $\left[\phi, C_{\lambda}\right]$, we define for every interval $I$ the following two numbers: 
(i) $L(\phi, I)=\lim \inf \phi\left(\varepsilon_{\lambda} I\right)$ as $\left\|\varepsilon_{\lambda} I\right\| \rightarrow 0$,

(ii) $U(\phi, I)=\lim \sup \phi\left(\varepsilon_{\lambda} I\right)$ as $\left\|\varepsilon_{\lambda} I\right\| \rightarrow 0$,

and call these numbers, which are finite or infinite, the lower and upper integrals of $\left[\phi, C_{\lambda}\right]$ over the interval $I$ respectively. Given any interval $I$, any number $\lambda$ such that $0 \leqq \lambda<1$, and any number $\delta>0$, it is easily shown that there exists an $\varepsilon_{\lambda} I$ such that $\left\|\varepsilon_{\lambda} I\right\|<\delta$. Consequently the lower and upper integrals are defined for every $I \in C_{0}$. In our bracket notation these functions (not necessarily finite) may be denoted by $\left[L(\phi), C_{0}\right]$ and $\left[U(\phi), C_{0}\right]$ respectively. In case $L(\phi, I)=U(\phi, I)$ is a finite number, we denote the common value by $F(\phi, I)$ and call it the integral of $\phi$ over $I$. Defining the integral in this manner, that is, for a function of intervals defined on a class $C_{\lambda}$, makes it sufficiently flexible to include the integral in the extended sense of Burkill, the strong integral of Saks, and the regular integral of Kempisty, by suitably choosing $\lambda\left({ }^{2}\right)$. If $\left[\phi, C_{\lambda}\right]$ is integrable over $R_{0}$ then it is integrable over every $I \in C_{0}$. The indefinite integral, which we may denote by $\left[F(\phi), C_{0}\right]$, is an additive function of intervals.

A function of intervals $\left[\phi, C_{0}\right]$ is said to be absolutely continuous in the restricted sense, briefly $R A C$, if the function $\left[U(|\phi|), C_{0}\right]$ is continuous.

1.5. The following theorem was stated and proved by Burkill [2, p. 289] for an integral which, under the stated assumptions, reduces to the integral as defined in 1.4 if $\lambda=0$.

THEOREM. If a function of intervals $\left[\phi, C_{\lambda}\right]$ is absolutely continuous and integrable, if $E$ is a measurable set, and if $\epsilon_{n}, n=1,2, \cdots$, is any decreasing sequence of positive numbers approaching 0 , and corresponding to each $n, E$ is decomposed into $\varepsilon_{n}+e_{n}^{\prime}-e_{n}^{\prime \prime}$ where $e_{n}^{\prime}$ and $e_{n}^{\prime \prime}$ are measurable sets such that $\left|e_{n}^{\prime}\right|$ and $\left|e_{n}^{\prime \prime}\right|$ are each less than $\epsilon_{n}$, and $\varepsilon_{n}$ is an elementary system of intervals, then as $n \rightarrow \infty, F\left(\phi, \varepsilon_{n}\right)$ approaches a limit which we call $F(E)$ and which is independent of the particular decomposition of $E$ for any $n$.

Burkill showed that the function $F(E)$, which is defined for all measurable subsets of $R_{0}$, is an absolutely continuous, completely additive function of measurable sets, which for intervals reduces to the integral. This is a strong type of extension in the sense that if any interval $I$ is given, and if $E$ is any set satisfying the relation $I^{0} \subset E C I$, then $F(E)=F(\phi, I)$. This property is a direct implication of the absolute continuity and additivity of the function $\left[F(\phi), C_{0}\right]$.

1.6. Burkill's result suggested the following type of extension. A completely additive set function $[\Phi, B]$ defined for all Borel sets in $R_{0}$ (briefly, an additive function of Borel sets) is a B-extension of a function $\left[\phi, C_{\lambda}\right]$ if $\Phi(E)=\phi(I)$ for every $I \in C_{\lambda}$ and for every Borel set $E$ such that $I^{\circ} \subset E \subset I$. In Part 3 we shall establish the following theorems:

(2) For Burkill's definition, see [2, p. 279]; for Saks's definition, see [11, p. 212]; for Kempisty's definition, see $[6$, p. 212$]$. 
THEOREM 4. A necessary and sufficient condition that a non-negative function of intervals $\left[\phi, C_{\lambda}\right]$ admit a non-negative $B$-extension is that it be an additive, continuous function.

THEOREM 5. A necessary and sufficient condition that a function of intervals $\left[\phi, C_{\lambda}\right]$ admit a B-extension is that it be additive and $R A C$.

THEOREM 6. A necessary and sufficient condition that the indefinite integral of an integrable function of intervals $\left[\phi, C_{\lambda}\right]$ admit a $B$-extension is that $\left[\phi, C_{\lambda}\right]$ be $R A C$.

\section{Completely additive extensions of functions of intervals.}

2.1. The necessity of condition $\mathfrak{C}$ in Theorem 1 is an immediate consequence of the following property of a completely additive set function: If $[\Phi, K]$ is any non-negative completely additive set function, if $\left\{e_{n}\right\}$ is any sequence of mutually exclusive sets in $K$, and if $\left\{E_{m}\right\}$ is any sequence of sets in $K$ such that $\sum_{m} E_{m} \supset \sum_{n} e_{n}$ then $\sum_{m} \Phi\left(E_{m}\right) \geqq \sum_{n} \Phi\left(e_{n}\right)$.

2.2. Let $\left[\phi, C_{\lambda}\right]$ be a non-negative function of intervals which satisfies condition $\mathfrak{C}$. For every set $E \subset R_{0}$ we define

$$
\phi(E)=\text { g.l.b. } \sum_{n} \phi\left(I_{\lambda n}\right)
$$

for all sequences $\left\{I_{\lambda_{n}}\right\}$ such that $\sum_{n} I_{\lambda_{n}} \supset E$. Obviously $\phi(E)$ is a non-negative function. We shall show that it is an outer measure in the sense of Carathéodory (see [12, p. 43]), that is, we shall show that it satisfies the following conditions :

(i) $\Phi\left(E_{1}\right) \leqq \Phi\left(E_{2}\right)$ whenever $E_{1} \subset E_{2}$.

(ii) $\bar{\phi}\left(\sum_{n} E_{n}\right) \leqq \sum_{n} \phi\left(E_{n}\right)$ for every sequence $\left\{E_{n}\right\}$ of sets.

(iii) $\bar{\phi}\left(E_{1}+E_{2}\right)=\bar{\phi}\left(E_{1}\right)+\bar{\phi}\left(E_{2}\right)$ whenever the distance from $E_{1}$ to $E_{2}$, which we denote by $d\left(E_{1}, E_{2}\right)$, is greater than 0 . Conditions (i) and (ii) follow directly from the definition of $\Phi(E)$ and from condition $(S$. We proceed to establish condition (iii).

A transversal of $R_{0}$, denoted generically by $t$, is a closed line segment which is parallel to either the $x$-axis (a horizontal transversal) or to the $y$-axis (a vertical transversal), and which satisfies the following two conditions: (i) the end points of $t$ lie on the boundary of $R_{0}$, (ii) the set which consists of $t$ less its end points-denoted by $t^{0}$-is contained in $R_{0}^{0}$.

Given a yy interval $I \in C_{0}$ we say that a subdivision $\varepsilon_{\lambda} I$ is a $\phi^{\prime}$-subdivision of $I$ if the set of intervals in $\varepsilon_{\lambda} I$ can be segregated into two sets, say $I_{1}, I_{2}, \cdots, I_{n}$ and $J_{1}, J_{2}, \cdots, J_{m}$, such that $I_{i} \cdot I_{j}=0$ for $i \neq j$ and $J_{i} \cdot J_{j}=0$ for $i \neq j$. Given a transversal $t$, it may be verified that there exist intervals $I$ such that $I \supset t, I^{0} \supset t^{0}$, and such that $I$ has a $\phi^{\prime}$-subdivision. A $\phi^{\prime}$-subdivision of an interval $I$, where $I \supset t, I^{0} \supset t^{0}$, is called a $\phi^{\prime}$-covering of $t$. For every transversal $t$ we define $\phi^{\prime}(t)=$ g.l.b. $\phi\left(\varepsilon_{\lambda} I\right)$ for all elementary systems $\varepsilon_{\lambda} I$ 
which are $\phi^{\prime}$-coverings of $t$. It follows from condition $(5)$ that there are at most a denumerable number of transversals $t$ for which $\phi^{\prime}(t)>0$.

Let $I_{0}$ be any interval in $C_{\lambda}$; let $t_{1}, t_{2}, \cdots, t_{n}$ be a finite number of horizontal transversals and $t_{n+1}, \cdots, t_{m}$ a finite number of vertical transversals such that $t_{i} \cdot I_{0}^{0} \neq 0$ for $i=1, \cdots, m$. These transversals determine a subdivision of $I_{0}$ into $(n+1)(m+1)$ intervals, say $I_{1}, I_{2}, \cdots, I_{k}$. We shall say that this elementary system is a regular subdivision of $I_{0}$ if $\phi^{\prime}\left(t_{i}\right)=0$ for $i=1,2, \cdots, m$, and $I_{i} \in C_{\lambda}$ for $i=1,2, \cdots, k$. Given a number $\delta>0$ it may be shown that there exists a regular subdivision of $I_{0}$-say $\varepsilon I_{0}$-such that $\left\|\varepsilon_{I_{0}}\right\|<\delta$.

Let $I$ be one of the intervals in a regular subdivision of an interval $I_{0} \in C_{\lambda}$. Let $I^{*}$ denote the set $I^{0}+b\left(I_{0}\right) \cdot I$. The set $I-I^{*}$ is the sum of $k, 1 \leqq k \leqq 4$, line segments. Let $t_{1}, t_{2}, \cdots, t_{k}$ be the set of transversals such that $t_{i}, i=1, \cdots, k$, contains one of the line segments in $I-I^{*}$. Given $\epsilon>0$ let $\varepsilon_{\lambda_{i}} J_{i}$ be a $\phi^{\prime}$-covering of $t_{i}$ such that $\phi\left(\varepsilon_{\lambda_{i}} J_{i}\right)<\epsilon / 4$. Let $J \in C_{\lambda}$ be an interval such that $I^{*} \supset J$ and $I \subset J+J_{1}+\cdots+J_{k}$. It follows from condition $\mathbb{E}$ that $\phi(I) \leqq \phi(J)+\phi\left(\varepsilon_{\lambda_{1} J_{1}}\right)+\cdots+\phi\left(\varepsilon_{\lambda_{k}} J_{k}\right)<\phi(J)+\epsilon$.

Let $I_{1}, I_{2}, \cdots, I_{k}$ be the intervals in a regular subdivision of $I_{0} \in C_{\lambda}$. Given $\epsilon>0$, let $J_{i}, i=1, \cdots, k$, be an interval in $C_{\lambda}$ such that $I_{i}^{*} \supset J_{i}$ and $\phi\left(I_{i}\right)<\phi\left(J_{i}\right)+\epsilon / k$. From condition $\mathbb{E}$ it follows that

$$
\phi\left(I_{0}\right) \leqq \sum_{i=1}^{k} \phi\left(I_{i}\right)<\sum_{i=1}^{k} \phi\left(J_{i}\right)+\epsilon \leqq \phi\left(I_{0}\right)+\epsilon .
$$

Since $\epsilon>0$ is arbitrary it follows that $\phi\left(I_{0}\right)=\phi\left(I_{1}\right)+\cdots+\phi\left(I_{k}\right)$. Thus $\left[\phi, C_{\lambda}\right]$ is additive over regular subdivisions.

Let $E$ be any set in $R_{0}$ and, given $\epsilon>0$, let $\left\{I_{n}\right\}$ be a sequence of intervals in $C_{\lambda}$ such that $\sum_{n} I_{n} \supset E$ and $\phi(E)>\sum_{n} \phi\left(I_{n}\right)-\epsilon$. Given $\delta>0$ let $\varepsilon_{\lambda_{n}} I_{n}$, $n=1,2, \cdots$, be a regular subdivision of $I_{n}$ such that $\left\|\varepsilon_{\lambda_{n}} I_{n}\right\|<\delta$. Arrange the set of intervals $J$ such that $J \in \varepsilon_{\lambda n} I_{i n}$ for some integer $n$ into a sequence $\left\{J_{m}\right\}$. Then $\sum_{m} J_{m} \supset E ; \phi(E)>\sum_{m} \phi(J)-\epsilon$; and $\left\|J_{m}\right\|<\delta$ for $m=1,2, \cdots$.

Let $E_{1}$ and $E_{2}$ be any two sets such that $d\left(E_{1}, E_{2}\right)=\delta>0$, and let there be given a number $\epsilon>0$. Let $\left\{I_{n}\right\}$ be a sequence of intervals in $C_{\lambda}$ such that $\sum_{n} I_{n} \supset E_{1}+E_{2},\left\|I_{n}\right\|<\delta, n=1,2, \cdots$, and $\phi\left(E_{1}+E_{2}\right)>\sum_{n} \phi\left(I_{n}\right)-\epsilon$. Let $I_{1 i}, i=1,2, \cdots$, be the intervals in $\left\{I_{n}\right\}$ such that $I_{1 i} \cdot E_{1} \neq 0$. Let $I_{2 j}$, $j=1,2, \cdots$, be the remainder of the intervals in $\left\{I_{n}\right\}$. Then $\sum_{i} I_{1 i} \supset E_{1}$; $\sum_{j} I_{2 j} \supset E_{2}$ and $\phi\left(E_{1}\right)+\bar{\phi}\left(E_{2}\right) \leqq \sum_{i} \phi\left(I_{1 i}\right)+\sum_{j} \phi\left(I_{2 j}\right)=\sum_{n} \phi\left(I_{n}\right)<\bar{\phi}\left(E_{1}+E_{2}\right)+\epsilon$. Since $\epsilon>0$ is arbitrary it follows that $\phi\left(E_{1}\right)+\Phi\left(E_{2}\right) \leqq \Phi\left(E_{1}+E_{2}\right)$. This result together with condition (ii) establishes condition (iii) in the definition of outer Carathéodory measure.

Applying the theory of outer Carathéodory measure (see [12, chap. 2]) we may now complete our proof of Theorem 1 . A set $E$ is $\phi$-measurable if $\Phi(P+Q)=\Phi(P)+\phi(Q)$ for every pair of sets $P$ and $Q$ contained, respectively, in the set $E$ and in its complement $C E$. The class of all sets which are $\phi$-meas- 
urable - we denote it by $K_{\phi}$-is an additive class in the sense of Saks, that is, (i) it contains the empty set, (ii) if $E \in K_{\phi}$ then $C E \in K_{\phi}$, and (iii) if $\left\{E_{n}\right\}$ is a sequence of sets in $K_{\phi}$, then $\sum_{n} E_{n}$ is a set in $K_{\phi}$. Furthermore, the class $K_{\phi}$ contains all sets which are open relative to $R_{0}$. Thus $K_{\phi}$ is a closed class of sets (1.2). The function $\left[\phi, K_{\phi}\right]$ is a completely additive set function; it is non-negative; and it follows from condition (5) that $\phi(I)=\phi(I)$ for every $I \in C_{\lambda}$. Thus $\left[\bar{\phi}, K_{\phi}\right]$ is a non-negative completely additive extension of the function $\left[\phi, C_{\lambda}\right]$. This completes a proof of Theorem 1 ; we proceed to outline a proof of Theorem 2.

2.3. Let $\left[\phi, C_{\lambda}\right]$ be a function of intervals and let $[\Phi, K]$ be a completely additive extension of $\left[\phi, C_{\lambda}\right]$. Then $[\Phi, K]$ is the difference of two non-negative completely additive set functions (see $\left[13\right.$, p. 90]), call them $\left[\Phi_{1}, K\right]$ and $\left[\Phi_{2}, K\right]$. We suppose that $\Phi(E)=\Phi_{1}(E)-\Phi_{2}(E)$ for every set $E \in K$. Each of the functions $\left[\Phi_{i}, K\right], i=1,2$, satisfies the condition stated in 2.1 ; in particular, each of the functions $\left[\Phi_{i}, C_{\lambda}\right]$ satisfies condition $\mathfrak{E}$. But $\phi\left(I_{\lambda}\right)=\Phi\left(I_{\lambda}\right)=\Phi_{1}\left(I_{\lambda}\right)-\Phi_{2}\left(I_{\lambda}\right)$; thus the condition in Theorem 2 is necessary.

Let $\left[\phi, C_{\lambda}\right],\left[\phi_{1}, C_{\lambda}\right],\left[\phi_{2}, C_{\lambda}\right]$ be finite, single-valued functions of intervals. We assume that $\left[\phi_{1}, C_{\lambda}\right]$ and $\left[\phi_{2}, C_{\lambda}\right]$ are non-negative functions each of which satisfies condition $\left(\mathcal{S}\right.$ and that $\phi(I)=\phi_{1}(I)-\phi_{2}(I)$ for every $I \in C_{\lambda}$. Let $\left[\Phi_{1}, K_{1}\right]$ and $\left[\Phi_{2}, K_{2}\right]$ be non-negative completely additive extensions of $\left[\phi_{1}, C_{\lambda}\right]$ and $\left[\phi_{2}, C_{\lambda}\right]$ respectively. Let $K$ denote the closed class $K_{1} \cdot K_{2}$. For every set $E \in K$ we define $\Phi(E)=\Phi_{1}(E)-\Phi_{2}(E)$. The function $[\Phi, K]$ is completely additive, and furthermore, the relation

$$
\phi(I)=\phi_{1}(I)-\phi_{2}(I)=\Phi_{1}(I)-\Phi_{2}(I)=\Phi(I)
$$

holds for every $I \in C_{\lambda}$. Thus $[\Phi, K]$ is a completely additive extension of $\left[\phi, C_{\lambda}\right]$. This establishes the sufficiency of the condition in Theorem 2.

$2.4\left(^{3}\right)$. Let $[\Phi, B]$ be a non-negative additive function of Borel sets (1.6). For every set $E \in B$ define $\phi(E)=$ g.l.b. $\sum_{n} \Phi\left(I_{n}\right)$ for all sequences $\left\{I_{n}\right\}$ of intervals in $C_{\lambda}$ such that $\sum_{n} I_{n} \supset E$. The function $[\Phi, B]$ is a non-negative completely additive extension of the function $\left[\Phi, C_{\lambda}\right]$. Therefore $\left[\Phi, C_{\lambda}\right]$ satisfies condition $\mathbb{E}$ and the function $\Phi(E)$ is completely additive on the class of all Borel sets. It follows from the definition of $\phi(E)$ and from condition (5) that $\phi\left(R_{0}\right)=\Phi\left(R_{0}\right)$ and that $\phi(E) \geqq \Phi(E)$ for every $E \in B$. Thus, for $E \in B$, we have $\Phi(E) \geqq \Phi(E), \quad \phi(C E) \geqq \Phi(C E)$, and $\phi(E)+\phi(C E)=\Phi\left(R_{0}\right)$ $=\Phi\left(R_{0}\right)=\Phi(E)+\Phi(C E)$. Obviously then, $\Phi(E)=\Phi(E)$.

2.5. Let $\left[\Phi_{1}, B\right]$ and $\left[\Phi_{2}, B\right]$ be any two non-negative additive functions of Borel sets and suppose that $\Phi_{1}(I) \geqq \Phi_{2}(I)$ for every $I \in C_{\lambda}$. For $E \in B$ and for $i=1,2$, define $\bar{\phi}_{i}(E)=$ g.l.b. $\sum_{n} \Phi_{i}\left(I_{n}\right)$ for all sequences $\left\{I_{n}\right\}$ such that $\sum_{n} I_{n} \supset E$. From 2.4 it follows that $\Phi_{i}(E)=\Phi_{i}(E)$ for every $E \in B$. But $\bar{\phi}_{1}(E) \geqq \Phi_{2}(E)$, and hence $\Phi_{1}(E) \geqq \Phi_{2}(E)$.

(3) The proof of Theorem 3 as presented here in $\$ \S 2.4-2.7$ was suggested by Professor Earl Mickle. For another treatment of the uniqueness of a completely additive extension, see [9]. 
2.6. Let $\left[\Phi_{1}, B\right]$ and $\left[\Phi_{2}, B\right]$ be any two additive functions of Borel sets such that $\Phi_{1}\left(I_{\lambda}\right) \geqq \Phi_{2}\left(I_{\lambda}\right)$ for every $I_{\lambda} \in C_{\lambda}$. We express each of the functions $\Phi_{1}, \Phi_{2}$ as the difference of two non-negative additive functions of Borel sets:

$$
\Phi_{1}(E)=\Phi_{11}(E)-\Phi_{12}(E) ; \quad \Phi_{2}(E)=\Phi_{21}(E)-\Phi_{22}(E) .
$$

Then for $I \in C_{\lambda}$ we have $\Phi_{11}(I)-\Phi_{12}(I)=\Phi_{1}(I) \geqq \Phi_{2}(I)=\Phi_{21}(I)-\Phi_{22}(I)$ and $\Phi_{11}(I)+\Phi_{22}(I) \geqq \Phi_{12}(I)+\Phi_{21}(I)$. From 2.5 it follows that if $E \in B$ then $\Phi_{11}(E)+\Phi_{22}(E) \geqq \Phi_{12}(E)+\Phi_{21}(E)$. From this it follows immediately that $\Phi_{1}(E) \geqq \Phi_{2}(E)$.

2.7. Let $\left[\Phi_{1}, K\right]$ and $\left[\Phi_{2}, K\right]$ be two completely additive extensions of a function $\left[\phi, C_{\lambda}\right]$. Then $\Phi_{1}(I)=\Phi_{2}(I)=\phi(I)$ for every $I \in C_{\lambda}$. It follows from 2.6 that $\Phi_{1}(E)=\Phi_{2}(E)$ for every $E \in B$. In other words, if $[\Phi, K]$ is a completely additive extension of a function $\left[\phi, C_{\lambda}\right]$, and if $E \in B$, then the value of $\Phi(E)$ is uniquely determined by the function $\left[\phi, C_{\lambda}\right]$. If $\left[\phi, C_{\lambda}\right]$ is nonnegative, the number $\Phi(E)$ has the characterization as defined in 2.2. Thus we have established Theorem 3 .

3. $B$-extensions. This part contains our results on the $B$-extension as stated in $\$ 1.6$. In $\$ \S 3.1$ and 3.2 we state and prove two lemmas which are used in the proofs of the main theorems.

3.1. Let $[\Phi, B]$ be a completely additive extension of a function of intervals $\left[\phi, C_{\lambda}\right]$. A necessary and sufficient condition that $[\Phi, B]$ be a $B$-extension of $\left[\phi, C_{\lambda}\right]$ is that $\Phi(I)=\Phi\left(I^{0}\right)$ for every $I \in C_{0}$.

Proof. Let $[\Phi, B]$ be a $B$-extension of $\left[\phi, C_{\lambda}\right]$. Then $\Phi(I)=\Phi\left(I^{0}\right)$ for every $I \in C_{\lambda}$. Let $I \in C_{0}$ and let $\varepsilon_{\lambda}$ be a subdivision of $I$ into the intervals $I_{1}, \cdots, I_{n}$. Express the set $I-I^{0}$ as the sum of mutually exclusive Borel sets $E_{1}+E_{2}+\cdots+E_{n}$ where $E_{i} \subset I_{i}-I_{i}^{0} ; i=1, \cdots, n$. Since $\Phi\left(E_{i}\right)=0$ it follows that $\Phi\left(I-I^{0}\right)=0$ and that $\Phi(I)=\Phi\left(I^{0}\right)$.

Let $[\Phi, K]$ be a completely additive extension of $\left[\phi, C_{\lambda}\right]$ and assume that $\Phi(I)=\Phi\left(I^{0}\right)$ for every $I \in C_{0}$. Let $i, i \subset R_{0}$, be any closed linear interval which is parallel to either the $x$ - or the $y$-axis. Let $I_{1} \supset I_{2} \supset \ldots$ be a descending sequence of intervals in $C_{0}$ such that $\prod_{n} I_{n}=i$ and $\prod_{n} I_{n}^{0}$ is the empty set. It follows from the additivity of $[\Phi, B]$ that

$$
0=\lim _{n} \Phi\left(I_{n}^{0}\right)=\lim _{n} \Phi\left(I_{n}\right)=\Phi(i) .
$$

Let $t$ be a transversal or a boundary segment of $R_{0}$. The function $\Phi(E)$ where $E \in B, E \subset t$ is a completely additive extension of the function of linear intervals $\Phi(i)$ where $i \subset t$. But such an extension is unique on Borel sets. Therefore, since $\Phi(i)=0$, it follows that $\Phi(E)=0$ for $E \in B, E \subset t$. Let $I$ be any interval in $C_{\lambda}$ and let $E \in B$ be any set such that $I^{\circ} \subset E \subset I$. Then $\Phi(I-E)=0$ and it follows that

$$
\phi(I)=\Phi(I)=\Phi(I-E)+\Phi(E)=\Phi(E) .
$$


Thus $[\Phi, B]$ is a $B$-extension of $\left[\phi, C_{\lambda}\right]$.

3.2. If a function of intervals $\left[\phi, C_{\lambda}\right]$ admits a $B$-extension, then $\left[\phi, C_{\lambda}\right]$ is additive.

Proof. Let $[\Phi, B]$ be a $B$-extension of $\left[\phi, C_{\lambda}\right]$. Let $I_{\lambda}$ and $\varepsilon_{\lambda} I_{\lambda}$ be given. Denote the intervals in $\varepsilon_{\lambda} I_{\lambda}$ by $I_{1}, I_{2}, \cdots, I_{n}$. Then

$$
\phi\left(I_{\lambda}\right)=\Phi\left(I_{\lambda}^{0}\right) \geqq \sum_{i=1}^{n} \Phi\left(I_{i}^{0}\right)=\sum_{i=1}^{n} \phi\left(I_{i}\right) .
$$

But $\sum_{i=1}^{n} I_{i} \supset I_{\lambda}$. It follows from condition $\mathbb{E}$ that $\phi\left(I_{\lambda}\right) \leqq \sum_{i=1}^{n} \phi\left(I_{i}\right)$. Thus $\left[\phi, C_{\lambda}\right]$ is additive. If we extend the range of definition of $\left[\phi, C_{\lambda}\right]$ from $C_{\lambda}$ to $C_{0}$ by defining $\phi(I)=\Phi(I)$, then the function $\left[\phi, C_{0}\right]$ is also additive.

3.3. We proceed to a proof of Theorem 4. Let $\left[\phi, C_{\lambda}\right]$ be a non-negative function of intervals and let $[\Phi, B]$ be a non-negative $B$-extension of $\left[\phi, C_{\lambda}\right]$. Extend the range of definition of $\left[\phi, C_{\lambda}\right]$ from $C_{\lambda}$ to $C_{0}$ by defining $\phi(I)=\Phi(I)$ for all $I \in C_{0}$. Let $R_{*}$ be a fixed interval such that $R_{*}^{0} \supset R_{0}$. Let $C_{*}$ denote the class of all intervals $I \subset R_{*}$. Define the function $\left[\phi_{*}, C_{*}\right]$ by the relation $\phi_{*}(I)=\phi\left(I \cdot R_{0}\right)$ if $I \cdot R_{0}$ is an interval in $C_{0}$; by the relation $\phi_{*}(I)=0$ for all other $I \in C_{*}$. Let $B_{*}$ denote the class of all Borel sets $E \subset R_{*}$ and define the function $\left[\Phi_{*}, B_{*}\right]$ by the relation $\Phi_{*}(E)=\Phi\left(E \cdot R_{0}\right)$ for every $E \in B_{*}$. Then $\left[\Phi_{*}, B_{*}\right]$ is a non-negative $B$-extension of $\left[\phi_{*}, C_{*}\right]$. Let $t$ be any transversal of $R_{0}$ or a closed boundary segment of $R_{0}$. Let $\left\{I_{n}\right\}$ be a sequence of intervals in $C_{*}$ such that $\prod_{n=1}^{\infty} I_{n}^{0}=t$. Then

$$
\lim _{n} \phi_{*}\left(I_{n}\right)=\lim _{n} \Phi_{*}\left(I_{n}\right)=\lim _{n} \Phi_{*}\left(I_{n}^{0}\right)=\Phi_{*}(t)=\Phi(t)=0 .
$$

Given $\epsilon>0$ let each vertical transversal of $R_{0}$ and each of the two vertical sides of $R_{0}$ be covered by an open interval $I^{0}$ such that $I \in C_{*}$ and $\phi_{*}(I)<\epsilon$. Then the closed set $R_{0}$ is covered by this class of open intervals. A finite number of these open intervals, say $I_{1}^{0}, I_{2}^{0}, \cdots, I_{n}^{0}$, suffice to cover $R_{0}$. Let $t_{1}, t_{2}$ denote the vertical boundary segments of $R_{0}$ and let $t_{3}, t_{4}, \cdots, t_{m}$ be the set of vertical transversals of $R_{0}$ which lie on the boundaries of the intervals $I_{i}, i=1, \cdots, n$. Let $\delta_{1}$ be the minimum of the numbers $d\left(t_{i} \cdot t_{j}\right)$ where $i \neq j$ and $i, j=1,2, \cdots, m$. Let $I \in C_{0}$ be any interval whose horizontal dimension is less than $\delta_{1}$. Then $I \subset I_{i}$ for some integer $i, i=1, \cdots, n$, and it follows that $\phi(I)=\phi_{*}(I) \leqq \phi_{*}\left(I_{i}\right)<\epsilon$. Similarly we may show that there exists a number $\delta_{2}>0$ such that if $I \in C_{0}$ is an interval whose vertical dimension is less than $\delta_{2}$ then $\phi(I)<\epsilon$. Let $\delta_{3}=\min \left(\delta_{1}, \delta_{2}\right)$, let $\delta$ be a number such that $0<\delta<\delta_{3}^{2}$, and let $I \in C_{0}$ be any interval such that $|I|<\delta$. Then at least one dimension of $I$ is less than $\delta_{3}$ and it follows that $\phi(I)<\epsilon$. Condition (ii) in the definition of continuity follows from the additivity of $\left[\phi, C_{\lambda}\right]$ which was established in 3.2. Thus the condition in Theorem 4 is necessary.

3.4. Let $\left[\phi, C_{\lambda}\right]$ be a non-negative, additive, continuous function of intervals. Let $I$ be any interval in $C_{0}$ and let $\varepsilon_{\lambda} I$ and $\mathcal{F}_{\lambda} I$ be any two subdivisions 
of $I$. Complete the elementary system $\varepsilon_{\lambda} I$ with an elementary system of intervals $G_{\lambda}$ to a subdivision of $R_{0}$. The system $\mathcal{F}_{\lambda}+G_{\lambda}$ also forms a subdivision of $R_{0}$ and we have that $\phi\left(\varepsilon_{\lambda}\right)+\phi\left(G_{\lambda}\right)=\phi\left(R_{0}\right)=\phi\left(\mathcal{F}_{\lambda}\right)+\phi\left(G_{\lambda}\right)$. Thus $\phi\left(\varepsilon_{\lambda}\right)=\phi\left(\mathcal{F}_{\lambda}\right)$. We extend the range of definition of $\left[\phi, C_{\lambda}\right]$ from $C_{\lambda}$ to $C_{0}$ by defining $\phi(I)=\phi\left(\varepsilon_{\lambda} I\right)$, where $\varepsilon_{\lambda} I$ is any subdivision of $I \in C_{0}$. The function $\left[\phi, C_{0}\right]$ is non-negative and additive. Using the definitional properties of continuity on the class $C_{\lambda}$, the additivity on the class $C_{0}$, and a technique similar to that employed in 3.3 , it may be proved that $\left[\phi, C_{0}\right]$ is a continuous function.

From the non-negative and additive properties of $\left[\phi, C_{0}\right]$ it follows that $\left[\phi, C_{0}\right]$ satisfies the following condition $\mathfrak{C}^{\prime}:$ If $I_{1}, I_{2}, \cdots, I_{n}$ is a finite set of intervals in $C_{0}$ such that $I_{i} \cdot I_{j}=0$ when $i \neq j$, and if $J_{1}, J_{2}, \cdots, J_{m}$ is a finite set of intervals in $C_{0}$ such that $\sum_{j=1}^{m} J_{j} \supset \sum_{i=1}^{n} I_{i}$, then $\sum_{j=1}^{m} \phi\left(J_{j}\right) \geqq \sum_{i=1}^{n} \phi\left(I_{i}\right)$.

We shall show that $\left[\phi, C_{0}\right]$ satisfies condition $\mathbb{E}$ as stated in Theorem 1. Let $R_{*}$ be a fixed interval such that $R_{*}^{0} \supset R_{0}$. Let the class $C_{*}$ and the function $\left[\phi_{*}, C_{*}\right]$ be defined as in 3.3. Then $\left[\phi_{*}, C_{*}\right]$ is a continuous, additive function. Let $I_{1}, I_{2}, \cdots, I_{n}$ be a finite set of mutually exclusive intervals-in $C_{0}$ and let $J_{1}, J_{2}, \cdots$ be an infinite sequence of intervals in $C_{0}$ such that $\sum_{j=1}^{\infty} J_{j} \supset \sum_{i=1}^{n} I_{i}$. Given $\epsilon>0$, let $R_{i}, i=1,2, \cdots$, be an interval in $C_{*}$ such that $R_{i}^{0} \supset J_{i}$ and $\phi_{*}\left(R_{i}\right)<\phi_{*}\left(J_{i}\right)+\epsilon / 2^{i}$. Then $\sum_{j=1}^{\infty} R_{j}^{0} \supset \sum_{i=1}^{n} I_{i}$. Since the set $\sum_{i=1}^{n} I_{i}$ is closed it follows that there is an integer $m$ such that $\sum_{j=1}^{m} R_{j}^{0} \supset \sum_{i=1}^{n} I_{i}$. Thus we have

$$
\sum_{i=1}^{n} \phi\left(I_{i}\right)=\sum_{i=1}^{n} \phi_{*}\left(I_{i}\right) \leqq \sum_{i=1}^{m} \phi_{*}\left(R_{i}\right)<\sum_{i=1}^{\infty} \phi_{*}\left(J_{i}\right)+\epsilon=\sum_{i=1}^{\infty} \phi\left(J_{i}\right)+\epsilon .
$$

Since $\epsilon>0$ is arbitrary, it follows that $\sum_{i=1}^{n} \phi\left(I_{i}\right) \leqq \sum_{j=1}^{\infty} \phi\left(J_{j}\right)$. Thus condition $\mathbb{S}^{\prime}$ is fulfilled when the finite set of $J$ 's is replaced by a sequence of $J$ 's. That it may be further extended by replacing the finite set of $I$ 's by a sequence of $I$ 's is obvious. Thus the function $\left[\phi, C_{0}\right]$ satisfies condition $\mathfrak{E}$. Let $[\Phi, B]$ be the non-negative completely additive extension of $\left[\phi, C_{0}\right]$ to the class $B$ : We shall show that $[\Phi, B]$ is a $B$-extension of $\left[\phi, C_{0}\right]$. Let $I$ be any interval in $C_{0}$. Given a number $\epsilon>0$, let $J \in C_{0}$ be an interval such that $J \subset I^{0}$ and $\phi(J)>\phi(I)-\epsilon$. Then

$$
\Phi(I)=\phi(I)<\phi(J)+\epsilon=\Phi(J)+\epsilon=\Phi\left(I^{0}\right)+\epsilon .
$$

Since $\epsilon>0$ is arbitrary, it follows that $\Phi(I)=\Phi\left(I^{0}\right)$ and from 3.1 it follows that $[\Phi, B]$ is a $B$-extension of $\left[\phi, C_{0}\right]$; obviously it is also a $B$-extension of the function $\left[\phi, C_{\lambda}\right]$. Thus the condition in Theorem 4 is sufficient.

3.5. Given a function of intervals $\left[\phi, C_{\lambda}\right]$ and a transversal $t$ of $R_{0}$ we define $\left.{ }^{4}\right)$ :

$A(t)=\lim \sup \left[\phi\left(\varepsilon_{\lambda}\right)-\phi\left(\mathcal{F}_{\lambda}\right)\right]$ for all $\varepsilon_{\lambda}$ and $\mathcal{F}_{\lambda}$ such that $\left\|\varepsilon_{\lambda}\right\| \rightarrow 0$,

( $)$ This definition is an extension of the concept of ecart as employed by Saks for functions of linear intervals. See $[11$, p. 211$]$. 
$\left\|\mathcal{F}_{\lambda}\right\| \rightarrow 0$, and $I \cdot t \neq 0$ for all $I \in \mathcal{E}_{\lambda}$ and $I \in \mathcal{F}_{\lambda}$.

$a(t)=\lim \inf \left[\phi\left(\mathcal{E}_{\lambda}\right)-\phi\left(\mathcal{F}_{\lambda}\right)\right]$ for all $\varepsilon_{\lambda}$ and $\mathcal{F}_{\lambda}$ as in the definition of $A(t)$.

The numbers $\Omega(t)=2^{-1}[A(t)+|A(t)|] \geqq 0$ and $\omega(t)=2^{-1}[a(t)-|a(t)|] \leqq 0$ are called the non-negative and non-positive ecarts of $\left[\phi, C_{\lambda}\right]$ on $t$. If both $\Omega(t)=0$ and $\omega(t)=0$, then $t$ is a transversal of zero ecart; otherwise $t$ is a transversal of nonzero ecart.

Let $\left[\phi, C_{\lambda}\right]$ be a function of intervals which is RAC, that is, the function $\left[U(|\phi|), C_{0}\right]$ is continuous. Let $t$ be a transversal of $R_{0}$. Given $\epsilon>0$, let $I$ be an interval in $C_{0}$ such that $I^{0} \supset t^{0}, I \supset t$, and $U(|\phi|, I)<\epsilon / 4$. Let $\eta>0$ be a number such that $\left\|\varepsilon_{\lambda} I\right\|<\eta$ implies $|\phi|\left(\varepsilon_{\lambda} I\right)<U(|\phi|, I)+\epsilon / 4$. Then if $\mathcal{F}_{\lambda_{1}}$ and $\mathcal{F}_{\lambda_{2}}$ are any two elementary systems such that $\left\|\mathcal{F}_{\lambda_{i}}\right\|<\eta, i=1,2$, and $J \subset I$ for every interval $J \in \mathcal{F}_{\lambda i}$, we have

$$
|\phi|\left(\mathcal{F}_{\lambda i}\right)<U(|\phi|, I)+\epsilon / 4<\epsilon / 2 .
$$

Thus $\left|\phi\left(\mathcal{F}_{\lambda_{1}}\right)-\phi\left(\mathcal{F}_{\lambda_{2}}\right)\right| \leqq|\phi|\left(\mathcal{F}_{\lambda_{1}}\right)+|\phi|\left(\mathcal{F}_{\lambda_{2}}\right)<\epsilon$. Since $\epsilon>0$ is arbitrary, it follows that $A(t)=a(t)=0$ and $R_{0}$ has no transversals of nonzero ecart.

3.6. If $\left[\phi, C_{\lambda}\right]$ increases (decreases) by subdivision and is RAC, then $U(\phi, I)(L(\phi, I))$ is finite and additive.

Proof. Since $\left[\phi, C_{\lambda}\right]$ increases by subdivision, it follows from a theorem of Kempisty that $U(\phi, I)>-\infty$ for all $I \in C_{0}$ and that $U(\phi, I)$ is additive. It follows from the continuity of $U(|\phi|, I)$ that $U(\phi, I)$ is also a continuous function. Let $\delta>0$ be a number such that $|I|<\delta$ implies $U(\phi, I)<1$. Let $\mathcal{E}_{I_{0}}$ be a subdivision of a fixed interval $I_{0}$ into intervals $I$ such that $|I|<\delta$. It follows that $U\left(\phi, I_{0}\right)$ is less than the number of intervals in $\varepsilon_{I_{0}}$.

If $\left[\phi, C_{\lambda}\right]$ decreases by subdivision, the result follows as a corollary if we consider the function $\left[-\phi, C_{\lambda}\right]$.

3.7. If $\left[\phi, C_{\lambda}\right]$ is RAC and increases (decreases) by subdivision, then $\left[\phi, C_{\lambda}\right]$ is integrable.

Proof. Let $\left[\phi, C_{\lambda}\right]$ be an RAC function which increases by subdivision. Then by 3.6, $U\left(\phi, R_{0}\right)$ is a finite number. Given $\epsilon>0$, let $\varepsilon_{\lambda} R_{0}$ be a subdivision of $R_{0}$ such that $\phi\left(\varepsilon_{\lambda} R_{0}\right)>U\left(\phi, R_{0}\right)-\epsilon$. Let $\left\{\varepsilon_{\lambda_{m}} R_{0}\right\}$ be a sequence of subdivisions of $R_{0}$ such that $\left\|\varepsilon_{\lambda_{m}} R_{0}\right\| \rightarrow_{m} 0$ and $\phi\left(\varepsilon_{\lambda_{m}} R_{0}\right) \rightarrow_{m} L\left(\phi, R_{0}\right)$. Let $t_{1}, t_{2}, \cdots, t_{k}$ be the set of all transversals of $R_{0}$ such that $t_{i}, i=1, \cdots k$, contains at least one boundary segment of an interval $I \in \varepsilon_{\lambda} R_{0}$. Let $\mathcal{F}_{m}$, $m=1,2, \cdots$, be the subdivision of $R_{0}$ which is formed by the transversals $t_{i}, i=1,2, \cdots, k$, and all of the boundary segments of intervals $I \in \varepsilon_{\lambda m}$. Let $T=\sum_{i=1}^{k} t_{i}$ and let $\varepsilon_{\lambda m}^{T}$ be the elementary system consisting of all the intervals $I$ such that $I \in \mathcal{E}_{\lambda m}$ and $I \cdot T \neq 0$. Each interval $I$ in $\mathcal{F}_{m}$ which is in the class $C_{0}-C_{\lambda}$ is contained in an interval $I \in \varepsilon_{\lambda m}^{T}$. We replace each such interval $I \in \mathcal{F}_{m}$ by a subdivision $\mathfrak{H C}_{\lambda} I$ and denote the resulting elementary system by $\mathcal{F}_{\lambda m}$. Let $\mathcal{F}_{\lambda m}^{T}$ denote the elementary system of intervals $I$ such that $I \in \mathcal{F}_{\lambda m}$ and $I$ is contained in some interval $J \in \mathcal{E}_{m \lambda}^{T}$. The elementary systems $\varepsilon_{\lambda m}-\varepsilon_{\lambda m}^{T}$ and $\mathcal{F}_{\lambda m}-\mathcal{F}_{\lambda m}^{T}$ are identical. Therefore $\phi\left(\mathcal{E}_{\lambda m}\right)-\phi\left(\mathcal{F}_{\lambda m}\right)$ 
$=\phi\left(\varepsilon_{\lambda m}^{T}\right)-\phi\left(\mathcal{F}_{\lambda m}^{T}\right)$. It follows from 3.5 that $\lim _{m}\left[\phi\left(\mathcal{E}_{\lambda m}^{T}\right)-\phi\left(\mathcal{F}_{\lambda m}^{T}\right)\right]=0$. Thus $\lim _{m} \phi\left(\mathcal{F}_{\lambda m}\right)=\lim _{m} \phi\left(\varepsilon_{\lambda m}\right)=L\left(\phi, R_{0}\right)$. Since $\left[\phi, C_{\lambda}\right]$ increases by subdivision we have

$$
\phi\left(\mathcal{F}_{\lambda m}\right) \geqq \phi\left(\varepsilon_{\lambda}\right)>U\left(R_{0}\right)-\epsilon, \quad m=1,2, \cdots .
$$

Therefore $L\left(\phi, R_{0}\right)=\lim _{m} \phi\left(\mathcal{F}_{\lambda m}\right)>U\left(\phi, R_{0}\right)-\epsilon$ and it follows that $\left[\phi, C_{\lambda}\right]$ is integrable. The case in which $\left[\phi, C_{\lambda}\right]$ decreases by subdivision follows as an immediate corollary since the function $\left[-\phi, C_{\lambda}\right]$ increases by subdivision.

3.8. We proceed to the proof of Theorem 5. Let $[\Phi, B]$ be a $B$-extension of the function of intervals $\left[\phi, C_{\lambda}\right]$. Express the function $[\Phi, B]$ as the difference of two non-negative additive functions of Borel sets, say $\left[\Phi_{1}, B\right]$ and $\left[\Phi_{2}, B\right]$, where $\Phi(E)=\Phi_{1}(E)-\Phi_{2}(E), E \in B$. For $i=1,2$ and $I \in C_{0}$ define $\phi_{i}(I)=\Phi_{i}(I)$. Then $\left[\phi_{1}, C_{0}\right]$ and $\left[\phi_{2}, C_{0}\right]$ are non-negative additive functions of intervals, and $\left[\Phi_{1}, B\right],\left[\Phi_{2}, B\right]$ are non-negative $B$-extensions of $\left[\phi_{1}, C_{0}\right]$, $\left[\phi_{2}, C_{0}\right]$ respectively. It follows from Theorem 4 that $\left[\phi_{1}, C_{0}\right],\left[\phi_{2}, C_{0}\right]$ are continuous functions. Since $\left|\phi\left(I_{\lambda}\right)\right| \leqq \phi_{1}\left(I_{\lambda}\right)+\phi_{2}\left(I_{\lambda}\right)$ for every $I \in C_{\lambda}$ it follows that $U(|\phi|, I) \leqq U\left(\phi_{1}, I\right)+U\left(\phi_{2}, I\right)=\phi_{1}(I)+\phi_{2}(I)$ for every $I \in C_{0}$. Thus $U(|\phi|, I)$ is a continuous function, that is, the function $\left[\phi, C_{\lambda}\right]$ is RAC. The necessity of the condition in Theorem 5 follows from this result and 3.2.

Let $\left[\phi, C_{\lambda}\right]$ be an additive, RAC function of intervals. For every $I \in C_{\lambda}$ define $\phi_{1}(I)=2^{-1}[\phi(I)+|\phi(I)|], \phi_{2}(I)=2^{-1}[|\phi(I)|-\phi(I)]$. Then $\left[\phi_{1}, C_{\lambda}\right]$, $\left[\phi_{2}, C_{\lambda}\right]$ are non-negative functions and $\phi\left(I_{\lambda}\right)=\phi_{1}\left(I_{\lambda}\right)-\phi_{2}\left(I_{\lambda}\right)$ for every $I \in C_{\lambda}$. It is readily proved that $\left[\phi_{1}, C_{\lambda}\right],\left[\phi_{2}, C_{\lambda}\right]$ are RAC and increase by subdivision. It follows from 3.7 that they are integrable. Their indefinite integrals, denoted by $\left[F_{1}, C_{0}\right]$ and $\left[F_{2}, C_{0}\right]$ respectively, are continuous, additive functions. Let $\left[\Phi_{1}, B\right]$ and $\left[\Phi_{2}, B\right]$ denote the $B$-extensions of $\left[F_{1}, C_{0}\right]$ and $\left[F_{2}, C_{0}\right]$ respectively. For every $E \in B$ define $\Phi(E)=\Phi_{1}(E)-\Phi_{2}(E)$. For $I \in C_{\lambda}$ we have $\phi(I)=\phi_{1}(I)-\phi_{2}(I)=\Phi_{1}(I)-\Phi_{2}(I)=\Phi(I)$. For $I \in C_{0}$ we have $\Phi(I)=\Phi_{1}(I)-\Phi_{2}(I)=\Phi_{1}\left(I^{0}\right)-\Phi_{2}\left(I^{0}\right)=\Phi\left(I^{0}\right)$. It follows from 3.1 that $[\Phi, B]$ is a $B$-extension of $\left[\phi, C_{\lambda}\right]$.

3.9. If $\left[\phi, C_{\lambda}\right]$ is integrable, then $U(|\phi|, I)=U(|F(\phi)|, I)$ for every $I \in C_{0}$.

Proof. Given a number $\epsilon>0$ let $\delta>0$ be a number such that $\left\|\varepsilon_{\lambda}\right\|<\delta$ implies $\left|\phi\left(\varepsilon_{\lambda}\right)-F\left(\phi, \varepsilon_{\lambda}\right)\right|<\epsilon / 2$ (see [6, Theorem 3]). Given an interval $I_{0} \in C_{0}$ let $\varepsilon_{\lambda} I_{0}$ be a subdivision of $I_{0}$ which consists of the intervals $I_{1}, \cdots, I_{n}$, and which is such that $\left\|\varepsilon_{\lambda} I_{0}\right\|<\delta$. Let $\varepsilon_{1}$ consist of the intervals $I \in \varepsilon_{\lambda} I_{0}$ which satisfy the relation $\phi(I)-F(\phi, I) \geqq 0$ and let $\varepsilon_{2}$ consist of the intervals $I \in \mathcal{E}_{\lambda} I_{0}$ which satisfy the relation $\phi(I)-F(\phi, I)<0$. Then $\left|\phi\left(\varepsilon_{i}\right)-F\left(\phi, \varepsilon_{i}\right)\right|$ $<\epsilon / 2, i=1,2$, and $\sum_{i=1}^{n}\left|\phi\left(I_{i}\right)-F\left(\phi, I_{i}\right)\right|<\epsilon$. But $\left|F\left(\phi, I_{i}\right)\right| \leqq\left|\phi\left(I_{i}\right)\right|$ $+\left|F\left(\phi, I_{i}\right)-\phi\left(I_{i}\right)\right|$;

$$
\left|\phi\left(I_{i}\right)\right| \leqq\left|F\left(\phi, I_{i}\right)\right|+\left|F\left(\phi, I_{i}\right)-\phi\left(I_{i}\right)\right| .
$$

Therefore ||$F\left(\phi, I_{i}\right)|-| \phi\left(I_{i}\right)|| \leqq\left|F\left(\phi, I_{i}\right)-\phi\left(I_{i}\right)\right|$, and $\sum_{i=1}^{n}|| F\left(\phi, I_{i}\right) \mid$ 
$-\left|\phi\left(I_{i}\right)\right| \mid<\epsilon$. It follows that $U\left(|\phi|, I_{0}\right)=U\left(|F(\phi)|, I_{0}\right)$.

3.10. Since the integral of a function of intervals is additive, it follows from Theorem 5 that: $A$ necessary and sufficient condition that the integral of $a$ function of intervals admit a B-extension is that the integral be $R A C$. Theorem 6 follows immediately from 3.9.

\section{BIBLIOGRAPHY}

1. S. Banach, Sur une classe de fonctions d'ensemble, Fund. Math. vol. 6 (1924) pp. 170-188.

2. J. C. Burkill, Functions of intervals, Proc. London Math. Soc. (2) vol. 22 (1923) pp. 275310.

3. E. W. Hobson, Theory of functions of a real variable, vol. 1, Cambridge, 1927.

4. E. Kamke, Das Lebesguesche Integral, Berlin, 1925.

5. S. Kempisty, Fonctions d'intervalle non additives, Actualités Scientifiques et Industrielles, no. 824, Paris, 1939.

6. - Sur les fonctions absolument continues d'intervalle, Fund. Math. vol. 27 (1936) pp. 10-37.

7. H. Kestelman, Modern theories of integration, Oxford, 1937.

8. A. J. Maria, Functions of plurisegments, Trans. Amer. Math. Soc. vol. 28 (1926) pp. 448471.

9. J. Radon, Theorie und Anwendungen der absolut-additiven Mengenfunktionen, Sitzungsberichte der mathematisch-naturwissenschaftlichen Klasse der Kaiserlichen Akademie der Wissenschaften vol. 122 (1913) pp. 1295-1438.

10. P. Reichelderfer and L. Ringenberg, The extension of rectangle functions, Duke Math. J. vol. 8 (1941) pp. 231-242.

11. S. Saks, Sur les fonctions d'intervalle, Fund. Math. vol. 10.(1927) pp. 211-224.

12. - Theory of the integral, Warsaw, 1937.

13. C. de la Vallée-Poussin, Integrales de Lesbesgue, Fonctions d'Ensemble, Classes de Baire, 2d ed., Paris, 1934.

UNIVERSITY OF MARYLAND,

College Park, MD. 